\title{
BMJ Open Prospective observational study in two Dutch hospitals to assess the performance of inflammatory plasma markers to determine disease severity of viral respiratory tract infections in children
}

\author{
Inge $\mathrm{M} \mathrm{L}$ Ahout, ${ }^{1}$ Kim H Brand, ${ }^{2}$ Aldert Zomer, ${ }^{3}$ Wilhelma $\mathrm{H}$ van den Hurk, ${ }^{4}$ \\ Geurt Schilders, ${ }^{4}$ Marianne L Brouwer, ${ }^{5}$ Chris Neeleman, ${ }^{6}$ Ronald de Groot, ${ }^{1}$ \\ Gerben Ferwerda ${ }^{1}$
}

To cite: Ahout IML, Brand KH, Zomer A, et al. Prospective observational study in two Dutch hospitals to assess the performance of inflammatory plasma markers to determine disease severity of viral respiratory tract infections in children. BMJ Open 2017;7:e014596. doi:10.1136/ bmjopen-2016-014596

- Prepublication history and additional material for this paper are available online. To view these files please visit the journal online (http://dx.doi. org/10.1136/bmjopen-2016014596).

Received 20 0ctober 2016 Revised 27 April 2017 Accepted 24 May 2017

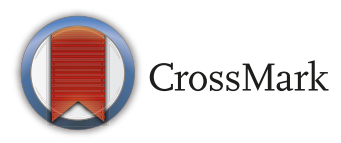

For numbered affiliations see end of article.

Correspondence to Dr Gerben Ferwerda; gerben. ferwerda@radboudumc.nl

\section{ABSTRACT \\ Introduction Respiratory viruses causing lower respiratory tract infections (LRTIs) are a major cause of hospital admissions in children. Since the course of these infections is unpredictable with potential fast deterioration into respiratory failure, infants are easily admitted to the hospital for observation. The aim of this study was to examine whether systemic inflammatory markers can be used to predict severity of disease in children with respiratory viral infections.}

Methods Blood and nasopharyngeal washings from children $<3$ years of age with viral LRTI attending a hospital were collected within 24 hours (acute) and after 4-6 weeks (recovery). Patients were assigned to a mild (observation only), moderate (supplemental oxygen and/or nasogastric feeding) or severe (mechanical ventilation) group. Linear regression analysis was used to design a prediction rule using plasma levels of $\mathrm{C}$ reactive protein (CRP), serum amyloid $A$ (SAA), pentraxin 3 (PTX3), serum amyloid P component and properdin. This rule was tested in a validation cohort.

Results One hundred and four children ( $52 \%$ male) were included. A combination of CRP, SAA, PTX3 and properdin was a better indicator of severe disease compared with any of the individual makers and age (69\% sensitivity (95\% Cl 50 to 83 ), $90 \%$ specificity $(95 \% \mathrm{Cl} 80$ to 96$))$. Validation in 141 patients resulted in $71 \%$ sensitivity ( $95 \%$ Cl 53 to 85 ), $87 \%$ specificity $(95 \% \mathrm{Cl} 79$ to 92$)$, negative predictive value of $64 \%$ (95\% Cl 47 to 78 ) and positive predictive value of $90 \%$ ( $95 \% \mathrm{Cl} 82$ to 95$)$. The prediction rule was not able to identify patients with a mild course of disease.

Conclusion A combination of CRP, SAA, PTX3 and properdin was able to identify children with a severe course of viral LRTI disease, even in children under 2 months of age. To assess the true impact on clinical management, these results should be validated in a prospective randomised control study.
Strengths and limitations of this study

- Two independent cohorts of young infants were used to identify and validate plasma biomarkers to assess severity of disease in children with viral lower respiratory tract infection (LRTI).

- Linear regression modelling was used to combine markers to optimise predictive value.

- Performance of our model was tested and validated in children under 2 months of age, the group that is clinically most relevant for severedisease.

- Other known inflammatory markers, such as procalcitonin, were not included and could have increased the clinical performance of test.

- This observational cohort study was performed during hospital admission of children with clinical signs of viral LRTI, validation in earlier stages of disease at GP office or ER would be more informative regarding clinical application.

\section{INTRODUCTION}

Acute lower respiratory tract infections (LRTIs) annually result in hospitalisation of $0.9 \%-1.36 \%$ of a birth cohort. ${ }^{12}$ Eventually, $6 \%$ to $15 \%$ of these patients are admitted to the intensive care unit (ICU). ${ }^{13}$ Young age and prematurity are the most important risk factors for severe diseases. This is reflected by the fact that over $50 \%$ of the children admitted to the ICU have no other risk factors besides young age. ${ }^{145}$

Infants under 2 to 3 months of age are easily admitted to the hospital due to a potentially rapid progression of disease. More than one-third of all patients hospitalised with acute respiratory viral infections do not receive supportive care. ${ }^{5}$ However, $5 \%$ of the 
patients discharged from the emergency room require readmission at a later stage of the infection. ${ }^{6}$ Therefore, there is a need for biomarkers that can predict the course of disease in infants with viral respiratory tract infections.

Currently, there are clinical prediction rules to predict safe discharge, length of hospitalisation and ICU admission for children with acute respiratory viral infections based on demographic criteria and clinical symptoms. ${ }^{45-11}$ Although these clinical prediction rules show the potential to improve clinical judgement, validation in other cohorts is often lacking or implementation in daily practice is difficult due to subjective criteria. ${ }^{89}$ Moreover, children under 2 months of age were either excluded or the studies were designed in such a way that all patients in this age group were automatically admitted to the hospital. A more objective, reproducible and sensitive prediction of disease severity for all age groups may be achieved by using biochemical and haematological markers. $^{12-16}$

Therefore, plasma proteins that were not yet extensively studied in the context of viral LRTI were identified from the literature. Pentraxin 3 (PTX3) has been described as an early sensitive and specific marker for bacterial pneumonia in bronchoalveolar lavages of mechanically ventilated adults, and as a severity marker of meningococcal disease in children. ${ }^{17}{ }^{18}$ In 2016, a study was published that showed that PTX3 levels are also a marker for LRTI in children and is more sensitive than $\mathrm{C}$ reactive protein (CRP), which is frequently used in clinical care. ${ }^{19}$ No correlation between CRP levels and severity of viral disease has been described. When CRP levels are very high, they indicate bacterial infection; however, often, CRP is moderately increased and then CRP is not able to differentiate between viral or bacterial aetiology of infection. ${ }^{20-23}$ Serum amyloid A (SAA) appears to be more sensitive than CRP for the detection of bacterial infections in neonates, although it is not used in daily practice. ${ }^{24}$ The SAA/CRP ratio can be used as a marker for severe disease in children with bacterial infections. ${ }^{25}$ Nakayama et al have shown that SAA levels increase in children with different viral infections, including viral LRTI. However, an association with disease severity was not established. ${ }^{26}$ Serum amyloid P component (SAP) can activate the classical complement pathway and interacts with mannan-binding lectin. SAP is part of a family of pattern recognition receptors, the pentraxin family. CRP and PTX3 belong to the same family. SAP is involved in inflammation and used as a marker for atherosclerosis and autoimmune disease. ${ }^{27}$ Skinner et al have shown that SAP levels were not increased during bacterial pneumonia, suggesting a limited potential as biomarker in infectious disease. ${ }^{28}$ No studies were found that assessed the role of SAP during viral infections; since its family members show potential as biomarker in viral disease, we decided to include SAP in this study.

Properdin (CFP) is a positive regulator of the alternative pathway of the complement system and it is a pattern recognition molecule that can bind to apoptotic/ necrotic cells or microbial pathogens (including viruses) to facilitate phagocytosis and clearance. ${ }^{29}{ }^{30}$ Recently, the immunological potential of CFP is gaining attention. In the context of kidney disease, the role of properdin is becoming more apparent; moreover it has been demonstrated that properdin can be activated via neutrophil extracellular traps. These traps are also present during viral LRTI and studied in the context of severe RSV infections. ${ }^{31}{ }^{32}$ Familial deficiencies in CFP are known and are associated with susceptibility to meningococcal infections. ${ }^{33}$ However, an increased susceptibility to viral infections has never been published.

In this study, we tested the aforementioned plasma proteins for their correlation with severity of viral LRTIs in children.

\section{MATERIALS AND METHODS}

\section{Study design}

Children younger than 3 years of age were eligible for inclusion in this prospective cohort study if they attended one of the two hospitals in Nijmegen from September until May with symptoms of an acute viral LRTI. Symptoms included signs of increased respiratory effort (eg, tachypnoea and/or use of accessory respiratory muscles or retractions) and/or expiratory wheezing and/or crackles on auscultation and/or apnoea. Patients with congenital or acquired immune deficiencies, immunosuppressive medication (including $>24$ hours of corticosteroids) or severe psychomotor retardation were excluded. Eligible patients were identified by their physician who diagnosed a viral LRTI and notified the study team. In both hospitals, it was not common practice to perform laboratory, rontgen or viral diagnostics at time of presentation; therefore, inclusions were made based on clinical appearance. When informed consent of both parents was obtained within 24 hours of admission, patients were included in the study. Data on clinical parameters, course of disease, medical history and demographics were retrieved from medical records and questionnaires retrieved at inclusion and after 4-6 weeks. Participation in this study did not influence medical care, and study results from individual patients were not shared with the medical team. Retrospectively, after discharge patients were allocated into three groups: mild, send home or only clinical observation; moderate, patients with need for supplemental oxygen and/or nasogastric feeding; and severe, patients with mechanical ventilation. Supplemental oxygen was started according to the protocols from the clinical wards in patients with an oxygen saturation of $\leq 92 \%$ for at least $10 \mathrm{~min}$ after use of decongestives. Results were evaluated in a training cohort ( $\mathrm{n}=104,2010-2012)$ and subsequently tested in a larger historical validation cohort with the same inclusion and exclusion criteria $(n=141,2006-2009)$. The study was approved by the Committee on Research involving Human Subjects. 


\section{Material collection}

After informed consent, blood and a nasopharyngeal wash were collected within 24 hours (acute), and after 4-6 weeks (recovery). A venous blood sample $(3 \mathrm{~mL})$ was collected in sodium heparin tubes and immediately transported to the laboratory. Plasma (and cell fractions) were obtained after density centrifugation (Lymphoprep, Axis Shield, Norway) and stored at $-80^{\circ} \mathrm{C}$. After instilling $0.5 \mathrm{~mL}$ of saline into one of the nostrils, a catheter was introduced into the nasopharynx to aspirate nasopharyngeal fluid. Samples were instantly put on ice, transported to the laboratory and stored at $-80^{\circ} \mathrm{C}$. Multiplex reverse transcription-PCR was performed on the nasal wash to confirm viral aetiology of disease, as previously described. ${ }^{35}$ This way, viral aetiology of disease was established for this study; however, this information was not available during treatment of the patient. The assay was designed to detect 15 viruses: influenza virus types A and B, coronavirus 229E and OC43, bocavirus, enterovirus, adenovirus, parechovirus, PIV types 1-4, metapneumovirus, rhinovirus and RSV.

\section{ELISA}

Levels of SAA, SAP, PTX3, CFP and CRP were determined in twofold diluted plasma by ELISA following the manufacturer's instructions (Hycult Biotech, Uden, The Netherlands, catalogue numbers HK333, HK331, HK347, HK334 and HK358, respectively).

\section{Statistics}

Data are expressed as percentages for categorical variables and as mean and SEM or median and IQR for the non-parametric continuous variables. Kruskal-Wallis tests were performed on continuous variables, followed by Mann-Whitney $\mathrm{U}$ test to further analyse differences. For categorical data, $\chi^{2}$ testing was used. Paired data were tested with the Wilcoxon signed-rank test. Correlations were calculated using Spearman's rho. The optimal predictive combination of proteins was selected using the M5 method linear regression, which removes classifiers with the smallest standardised coefficient one-by-one until no decrease in error estimate is observed. The outcome of the obtained rule was analysed for its receiver operating characteristics (ROCs). Cut-off values were determined for both mild and severe disease. These cut-offs were subsequently validated in a second cohort. A two-sided $p$ value of $<0.05$ was considered statistically significant. A subanalysis was performed in children under 2 months of age, since this group is often excluded in other studies that describe prediction rules and age is an important factor in disease severity. Statistical tests were performed by SPSS for Windows (Release 19, SPSS) and Waikato Environment for Knowledge Analysis was used to perform linear regression. ${ }^{36}$

\section{RESULTS \\ Patient characteristics}

The training cohort consisted of 104 children (overall inclusion percentage $58 \%$, off all patients/asked who were contacted informed consent was given in $77 \%$, see supplemental flowchart) (table 1). Children with severe disease were significantly younger and had more often siblings than patients in the mild and moderate groups. The amount of children below 2 months of age is 5, 12 and 21 in the mild, moderate and severe group, respectively. Duration of hospitalisation significantly increased towards more severe disease. Two per cent of the patients were not hospitalised $(2 / 104)$, whereas $17 \%$ of the hospitalised patients had only mild disease. RSV was detected in the majority of patients (65\%), in $43 \%$ viral coinfections were present. The highest proportion of RSV mono-infections was seen in children with a severe course of disease $(\mathrm{p}<0.001)$, as was previously published by our group. ${ }^{35}$

\section{Markers for infection and disease severity}

Plasma levels of CRP, SAP, SAA and PTX3 increased significantly during disease compared with levels in recovery samples, whereas CFP levels did not differ between these two time points (figure $1 \mathrm{~A}-\mathrm{E}$ ). When the acute disease group was subdivided into three severity classes, plasma levels of CRP during severe disease were significantly

Table 1 Patient characteristics

\begin{tabular}{|c|c|c|c|c|c|}
\hline & $\begin{array}{l}\text { Total } \\
n=104(\%)\end{array}$ & Mild $n=20(\%)$ & Moderate $n=52(\%)$ & Severe $n=32(\%)$ & p Value \\
\hline Gender (male) & $54(52 \%)$ & $12(60 \%)$ & $24(46 \%)$ & $18(56 \%)$ & NS \\
\hline Prematurity ( $<35$ weeks) & $5(5 \%)$ & $1(5 \%)$ & $3(6 \%)$ & $1(3 \%)$ & NS \\
\hline Onset symptoms (days) & $3.0(2.0-4.8)$ & $2.5(1.3-3.8)$ & $3.0(2.0-5.0)$ & $4.0(2.0-4.8)$ & NS \\
\hline$\%$ mono-infection RSV & $39(57 \%)$ & $3(37.5 \%)$ & $20(53 \%)$ & $16(70 \%)$ & $<0.001$ \\
\hline
\end{tabular}

Values are given in numbers (percentages) or medians (25th-75th percentiles). NS not signifficant.

*Mild versus severe $p=0.001$, moderate versus severe $p<0.0001$.

${ }^{* *}$ Mild versus moderate/severe $p<0.0001$, moderate versus severe $p<0.0001$

${ }^{* \star \star}$ Mild versus moderate/severe $(p<0.05)$. 

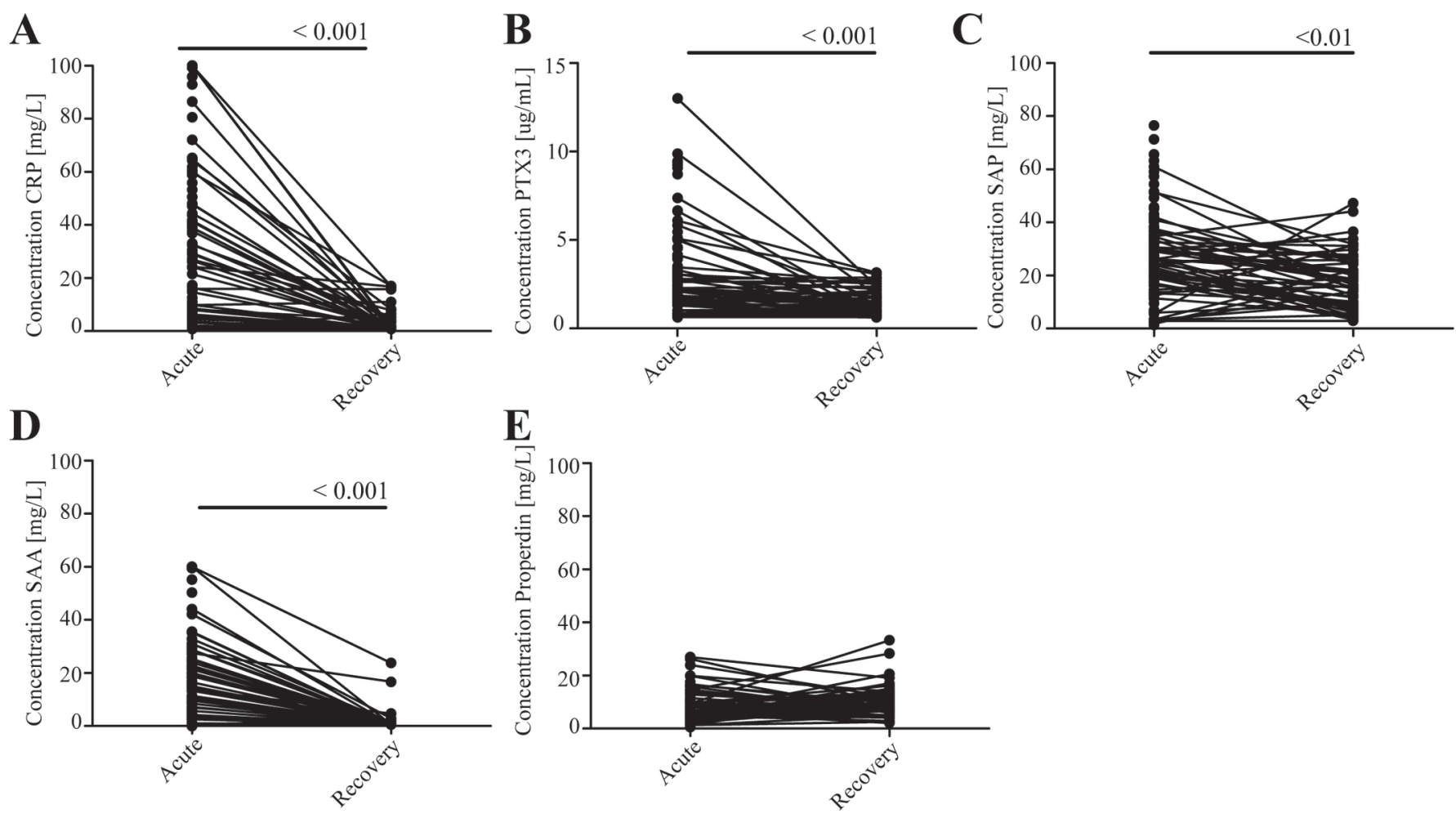

Figure 1 Comparison of plasma protein levels in children with acute respiratory viral infections with their paired recovery sample $(n=51)$ taken 4-6 weeks after acute infection is shown for CFP (E), SAA (D), SAP (C), C reactive protein (CRP) (A) and PTX3 (B) (Wilcoxon matched-pairs signed-rank test). CRP, C reactive protein; PTX3, pentraxin 3; SAA, serum amyloid A; SAP, serum amyloid $\mathrm{P}$ component.

higher compared with patients with mild and moderate disease (figure 2A). Although there was a stepwise incline in plasma levels of PTX3, there were no significant differences (figure 2B). Plasma levels of SAP were also equal between the different disease severities (figure 2C). SAA plasma levels, however, were significantly higher during severe disease compared with mild disease (figure 2D). CFP plasma levels did not differ between mild and moderate disease. In contrast to the other markers, the CFP plasma levels dropped significantly in patients with severe disease (figure 2E).

Since disease severity can also be reflected by the duration of hospitalisation and the need for supplemental oxygen (as substitute for the need for hospitalisation), we tested whether they correlated with the plasma proteins. Both CRP and CFP correlated significantly with duration of hospitalisation $(\rho=0.361, p \leq 0.001$ and $\rho=-0.22, p<0.05$, respectively). However, PTX3, SAP and SAA levels did not. The need for supplemental oxygen was associated with higher levels of CRP and PTX3 (figure 2A-B), whereas the other markers showed no significant difference.

\section{The influence of age and viral aetiology on protein levels}

Children with severe disease are significantly younger (table 1); therefore, we checked whether age is a confounder for our protein levels. After stratification of the cohort per severity and age group (under and above 2 months of age), protein levels were compared. After Bonferroni correction, no significant differences were found in the group under 2 months of age. Thus, although age is an important confounder for disease severity, the levels of the plasma markers are not only dependent on age and reflect disease severity.

RSV mono-infections are more frequently seen in the severe disease group (table 1). We tested whether patients with RSV mono-infections also had higher plasma levels compared with patients with the same disease severity without RSV mono-infections. We found that patients with RSV mono-infections had significantly higher SAA levels during severe disease $(\mathrm{p}<0.05)$. Levels of all other inflammatory markers were equal.

\section{Accuracy of the inflammatory markers to predict disease severity}

After finding differences in plasma levels in patients with different disease severities, the diagnostic performance of the proteins was tested. First, the performance of the markers to indicate the need for hospitalisation (supplemental oxygen and/or nasogastric feeding) was assessed. Of 104 patients included for analysis, 27 did not receive supplemental oxygen: six of these patients belonged to the moderate group (table 1). The area under the curve (AUC) for the markers CRP and PTX3 was 0.63 and 0.64, respectively. The AUC for the other markers was 0.59 for SAA, 0.57 for SAP and 0.51 for CFP.

Second, the performance of markers to indicate the need for mechanical ventilation (severe disease) was assessed. Of 104 patients included for analysis, 32 were 

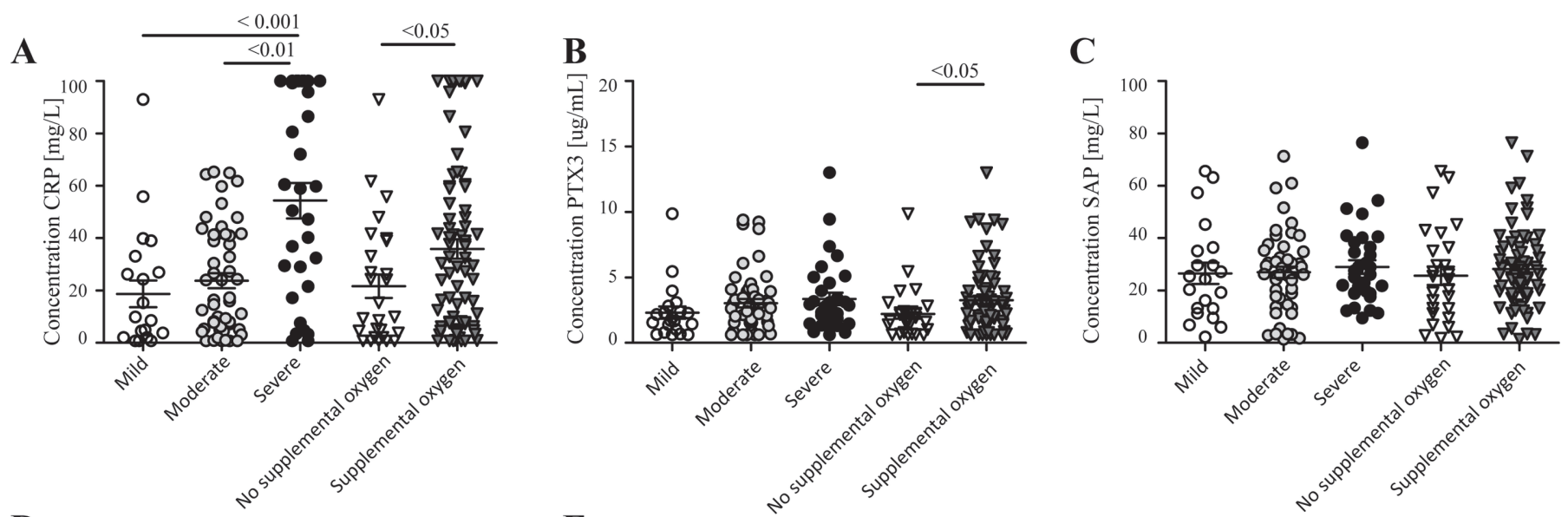

D

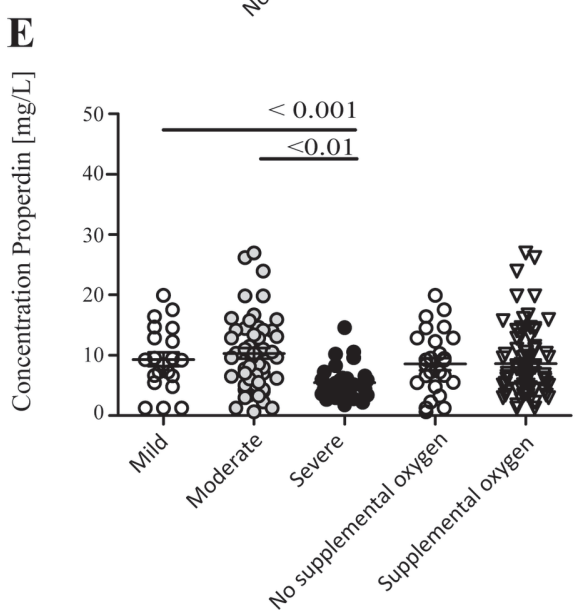

Figure 2 Comparison of protein levels between patients with mild $(n=21)$, moderate $(n=51)$ and severe $(n=32)$ disease and for patients with $(n=77)$ and without $(n=27)$ the need for supplemental oxygen are shown for $C$ reactive protein $(C R P)(A), P T X 3(B)$, SAP (C), SAA (D) and CFP (E) (Mann-Whitney $U$ test). CRP, C reactive protein; PTX3, pentraxin 3; SAA, serum amyloid A; SAP, serum amyloid $\mathrm{P}$ component.

mechanically ventilated (table 1). The AUC of the markers that were associated with severe disease, CRP, SAA and CFP, was $0.73,0.57$ and 0.76 , respectively. The AUC for the other markers was 0.56 for PTX3 and 0.53 for SAP.

\section{Combining inflammatory markers increases performance}

As reflected by the AUC, none of the single markers was considered sufficient to identify mild disease and thus aid in the decision to discharge a patient. Whereas the AUC of some markers for severe disease were slightly higher, none of them exceeded 0.8 .

Therefore, linear regression modelling was used to analyse whether a combination of markers could increase the individual performance. The assumption was that distances between the three groups were equal. The linear regression resulted in the following model: (5.8xPTX$3)+(1.15 \mathrm{xCRP})-(4.1 \mathrm{xCFP})-(0.93 \mathrm{xSAA})+106.25$. The AUC for this rule is 0.65 for mild disease (no need for supplemental oxygen) and 0.89 for severe disease (mechanical ventilation).

Based on the ROCs, a cut-off value for the identification of mild disease was set at 93.50, which resulted in an AUC of 0.65 and sensitivity of $56 \%$ (95\% CI 36 to 74), a specificity of $70 \%$ (95\% CI 58 to 80 ), a positive predictive value (PPV) of $82 \%$ (95\% CI 70 to 90) and a negative predictive value (NPV) of $39 \%$ (95\% CI 24 to 56). In $<2$ months old children, the AUC is 0.88 (sensitivity $87 \%$ (95\% CI 69 to 96 ), specificity $71 \%$ (95\% CI 30 to 95), PPV 93\% (95\% CI 76 to 99) and NPV 56\% (95\% CI 23 to $85)$ ), whereas in $>2$ months old patients, the AUC is 0.50 (sensitivity $57 \%$ (95\% CI 42 to 71 ), specificity $47 \%$ (95\% CI 25 to 71), PPV 72\% (95\% CI 56 to 96) and NPV 31\% (95\% CI 16 to 51$)$ ).

The cut-off value for severe disease was set at 122 (sensitivity $69 \%$ (95\% CI 50 to 83 ), specificity $90 \%$ (95\% CI 80 to 96 ) with a PPV of $76 \%$ (95\% CI 56 to 89) and an NPV of $87 \%$ ( $95 \%$ CI 76 to 93)) with an AUC of 0.88 . In children below 2 months of age, the AUC for severe disease increased to 0.90 (sensitivity $72 \%$ (95\% CI 50 to 87), specificity $85 \%$ (95\% CI 65 to 95 ), PPV 82\% (95\% CI 59 to 94) and NPV $77 \%$ (95\% CI 57 to 89)). In older children, the AUC is 0.86 (sensitivity $55 \%$ (95\% CI 25 to 82 ), specificity 93\% (95\% CI 82 to 98), PPV 60\% (95\% CI 27 to 86) and NPV $91 \%$ (95\% CI 80 to 97$)$ ).

\section{Validation of prediction rules}

A new cohort of 141 children was tested. Patient characteristics of the original and validation cohort were similar with respect to their distribution of age, gender and 
number of prematurely born infants (less than 35 weeks). The number of children below 2 months of age is 12, 23 and 22, respectively. In contrast to the training cohort, the presence of siblings was not significantly different between the severity groups in the validation cohort (see online supplementary table 1 and supplementary figure 1).

\section{Need for hospitalisation}

Validation of the prediction rule for mild disease, need for hospitalisation, resulted in 51\% sensitivity (95\% CI 37 to 65 ) and $68 \%$ specificity (95\% CI 58 to 78 ) (PPV $46 \%$ (95\% CI 33 to 60 ) and NPV $72 \%$ (95\% CI 62 to 81$)$ ). In children below 2 months of age, the rule performed better the AUC increased from 0.67 to 0.79 (sensitivity $55 \%$ (95\% CI 33 to 75 ), specificity $81 \%$ (95\% CI 70 to 89 ), PPV 46\% (95\% CI 27 to 66) and NPV 86\% (95\% CI 74 to 92)). In children $>2$ months, the AUC decreased to 0.58 (sensitivity $52 \%$ (95\% CI 38 to 65 ), specificity $60 \%(95 \%$ CI50 to 70 ), PPV $42 \%$ (95\% CI 30 to 55) and NPV $69 \%$ (95\% CI 58 to 78$)$ ).

\section{Need for mechanical ventilation}

When the prediction rule was applied to the validation set with a cut-off of 122 , the sensitivity for severe disease was $71 \%$ (95\% CI 53 to 85 ) with $87 \%$ specificity (95\% CI 79 to 92), PPV and NPV were $64 \%$ (95\% CI 47 to 78 ) and $90 \%$ (95\% CI 82 to 95$)$, respectively.

In children below 2 months of age, the AUC for severe disease is 0.87 (sensitivity 77\% (95\% CI 61 to 88), specificity 57\% (95\% CI 46 to 67), PPV 45\% (95\% CI 33 to 57), NPV $84 \%$ (95\% CI 73 to 92)). In older children, the AUC remains 0.87 (sensitivity 58\% (95\% CI 37 to 77 ), specificity $90 \%$ (95\% CI 83 to 94 ), PPV 52\% (95\% CI 32 to 71 ), NPV $92 \%$ (95\% CI 85 to 96)).

Additional analyses were performed to assess the performance of logistic regression analysis; this was not possible for mild disease since all patients were allocated to the mild/severe group. For severe disease, the performance of the rule was almost the same as compared with our linear regression model. The inclusion of age in the linear model resulted in a similar performance in the cohorts. In this rule, SAA was replaced with age.

\section{DISCUSSION}

In this study, we show that plasma levels of CRP, PTX3, SAA and CFP correlate with disease severity in children with acute viral LRTI. Furthermore, we demonstrate that a combination of these markers significantly increased the performance of the individual proteins to identify patients with severe disease. The designed prediction rule has been validated in a larger independent patient cohort with similar characteristics, which resulted in a comparable performance. Moreover, the performance of the rule was best in patients under 2 months of age $(<60.5$ days) in whom it is clinically difficult to predict the course of disease.
Interestingly, most of the inflammatory markers investigated in this study have not been described in the context of viral LRTI or disease severity. The increase of CRP and SAA levels during viral infection was known and this was also the reason to include the proteins in our study. We were able to confirm the work of Huttunen $e t$ al that the ratio SAA/CRP is significantly decreased in patients with severe disease compared with patients with mild and moderate disease (data not shown). ${ }^{25}$ Moreover, CRP and SAA levels both correlated with severity of viral LRTIs and CRP was also correlated with length of hospitalisation. To the best of our knowledge, these findings have not been described before. The increase in PTX3 levels during more severe disease and a correlation with need for supplemental oxygen show the potential of PTX3 as biomarker for viral LRTI in children. This was already proposed by others, who saw a correlation with peak temperature, duration of fever and presence of pneumonia in mechanically ventilated patients. ${ }^{17}{ }^{19}$ Although SAP levels significantly increase during infection, no correlation could be observed with disease severity in children with viral LRTI. Therefore, it appears that SAP could not be used as a biomarker for disease severity in viral or bacterial infections. ${ }^{28}$ This study is the first to describe the remarkable kinetics of CFP (properdin) levels, which decrease during severe viral infection in children. We were unable to find an explanation in the literature. However, since CFP levels restored in the available recovery samples, a deficiency is highly unlikely. Other possible explanations for the decrease might be consumption, as occurs with other complement factors, or an unrecognised bacterial coinfection. Although cultures were routinely taken at the intensive care unit before the start of selective digestive decontamination (SDD) antibiotics, bacterial infections cannot be ruled out completely. A subanalysis of patients with $(n=10)$ and without $(n=15)$ positive bacterial cultures on the ICU showed no difference in protein levels (data not shown). During the study period, SDD was standard practice in the ICU and, therefore, all severe patients would have had at least one gift of prophylactic antibiotics before the study samples were taken.

The prediction rule for the identification of children with a mild course of disease appeared to be insufficient for clinical use (AUC 0.65). However, the performance in children less than 2 months of age (AUC 0.88) is promising and might provide additive value in clinical decision-making. Currently, there are no comparable laboratory-based studies assessing the identification of mild disease. Clinical rules for safe discharge based on age and findings at physical examination have been developed. Unfortunately, in these studies, children below 2 months of age were excluded or automatically admitted regardless of the necessity of treatment. ${ }^{5333738}$

The identification of patients with severe disease was accurate with the chosen inflammatory markers (AUC $0.89)$. The prediction performed better than age on itself (AUC 0.77). A prediction rule based on interleukin- 8 and 
regulated on activation, normal $\mathrm{T}$ Cell expressed and secreted (RANTES) in plasma and $\mathrm{CD} 4^{+} \mathrm{T}$ cell counts accurately identifies severe disease but needs to be validated in an independent cohort to establish clinical potential. ${ }^{16}$ A combination of biochemical, haematological and clinical data might further improve accuracy. A drawback of introducing clinical variables is that most of the published risk factors in clinical prediction are not objective or may change rapidly in time. Moreover, signs and symptoms of children can be ambiguous at the moment of presentation and only become clear after prolonged observation. Prediction rules using clinical data often show a lower AUC and validation studies of these rules are lacking or have a smaller sample size. ${ }^{49}$

Limitations of our study are the limited number of patients in the mild age group, especially patients younger than 2 months of age. This influenced our analyses and hindered the logistic regression analysis in the prediction of mild disease. Since venapuncture is not always part of routine care, especially not in this patient group, parents often refuse participation or the venapuncture specific. Therefore, the performance of our rule in the mild patient group under 2 months of age needs further testing Moreover, a prospective study in which results are available for the clinicians is needed to assess the clinical implications of our rule.

Age is always a confounder in studies on disease severity in infants with RSV. We could not find correlations in the acute samples after correcting for age and multiple testing; however, we did observe a positive correlation between age and SAA and SAP in the recovery samples (data not shown). This is opposite to our findings during the acute phase of disease (higher levels in younger patients). Bruun $e t$ al studied plasma levels of CRP and SAA in children and neonates, including preterms in healthy and diseased state. They demonstrated that with increasing age (preterm up to child), higher CRP and SAA levels are being produced during disease. ${ }^{40}$ Although when patients were healthy, the differences resolve. It is likely that the higher levels of SAA in our youngest (severely ill) patients is a result of disease severity. This shas already been demonstrated for bacterial infections. ${ }^{24}{ }^{25}$ In our linear regression rule, SAA could be replaced with age of the patients which gave similar results.

It would be interesting to perform a study that assesses the plasma levels of our markers at different time points during viral infections to increase our insight in the kinetics. It is assumed that some biomarkers precede clinical symptoms and could therefore be used early in the course of disease to predict severe outcomes. The advantage of measuring markers in plasma is the possibility for easy implementation in current diagnostic laboratory settings, relative short time to measure, reproducibility of the assays and available options for standardisation. Moreover, with the use of tools for linear regression modelling, it is easy to add new markers to further optimise the predictive rule. In general, our results indicate that combining markers, in our case inflammatory proteins, could increase the performance of a test. This indicates that this method might also be useful in other settings. More specifically, due to the validation in an independent cohort, we can assume that our decision rule will perform as well in other hospital settings. Whether the combination of these plasma markers could also be used to predict severe bacterial infections need to be assessed in other studies.

Currently, there is no targeted therapy for most respiratory viruses and, therefore, there are no consequences to the early identification of patients with a severe course of disease besides optimisation of supportive care. The identification of children with a mild course of disease has more visible consequences, as can be measured in reduced hospitalisation rates or length of stay in the hospital. To truly estimate the value of the markers, a prospective study is needed. However, even before the markers are implemented in the clinic, biomarkers could be used to stratify patients in clinical studies and as secondary end-point in intervention studies. With the current development of point-of-care tests, plasma protein levels can be available within a relevant time frame and could be beneficial for care in emergency rooms and by the general practitioner.

\section{Author affiliations}

${ }^{1}$ Laboratory of Pediatric Infectious Diseases, Department of Pediatrics, Radboud Center for Infectious Diseases, Radboud university medical center, Nijmegen, The Netherlands

${ }^{2}$ Department of Pediatrics, Erasmus MC Sophia, Rotterdam, The Netherlands ${ }^{3}$ Department of Infectious Diseases and Immunology, Utrecht University, Utrecht, The Netherlands

${ }^{4}$ Department Research and Development, Hycult Biotech, Uden, The Netherlands ${ }^{5}$ Department of Pediatrics, Canisius Wilhelmina Hospital, Nijmegen, The Netherlands ${ }^{6}$ Department of Intensive Care Medicine, Radboud university medical center, Nijmegen, The Netherlands

Acknowledgements We would like to thank all parents and children who participated in this study. We are also thankful for the help of the staff from the participating hospitals. We thank Tefa for their Cheiron Dynamic II apparatus to enable us to perform nasal washes in a standardised matter and the Paediatric Drug Research Centre Nijmegen, especially Mariëtte Las, for excellent support during the study. Finally, we would like to thank Marloes Vissers for critically reading this manuscript.

Contributors $I A$ and $K B$ were involved in the design of the study, the inclusion of the cohorts. IA and AZ performed the analysis for this manuscript, WH and GS performed analysis, corrected and approved the manuscript. KB approved the manuscript. MB and $\mathrm{CN}$ were involved in the inclusion of the patients, corrected and approved the manuscript. RG and GF designed the whole project and helped in the design of the study, analysis and writing of the study. They approved the manuscript.

Funding IA, AZ, KB and GF are supported by the Virgo consortium, funded by the Dutch government project number FES0908, and by the Netherlands Genomics Initiative (NGI) project number 050-060-452. The funders had no role in study design, data collection and analysis, decision to publish or preparation of the manuscript.

Competing interests None declared.

Patient consent Informed consent was obtained from both parents / caregivers.

Ethics approval The Committee on Research involving Human Subjects Nijmegen.

Provenance and peer review Not commissioned; externally peer reviewed.

Data sharing statement Data are available on request via the corresponding author.

Open Access This is an 0pen Access article distributed in accordance with the Creative Commons Attribution Non Commercial (CC BY-NC 4.0) license, which permits others to distribute, remix, adapt, build upon this work non-commercially, 
and license their derivative works on different terms, provided the original work is properly cited and the use is non-commercial. See: http://creativecommons.org/ licenses/by-nc/4.0/

(C) Article author(s) (or their employer(s) unless otherwise stated in the text of the article) 2017. All rights reserved. No commercial use is permitted unless otherwise expressly granted.

\section{REFERENCES}

1. Berger TM, Aebi C, Duppenthaler A, et al. Prospective populationbased study of RSV-related intermediate care and intensive care unit admissions in Switzerland over a 4-year period (2001-2005). Infection 2009;37:109-16

2. Jansen AG, Sanders EA, Hoes AW, et al. Influenza- and respiratory syncytial virus-associated mortality and hospitalisations. Eur Respir $J$ 2007;30:1158-66.

3. Purcell K, Fergie J. Driscoll Children's Hospital respiratory syncytial virus database: risk factors, treatment and hospital course in 3308 infants and young children, 1991 to 2002. Pediatr Infect Dis J 2004;23:418-23.

4. Damore D, Mansbach JM, Clark S, et al. Prospective multicenter bronchiolitis study: predicting intensive care unit admissions. Acad Emerg Med 2008;15:887-94.

5. Mansbach JM, Clark S, Christopher NC, et al. Prospective multicenter study of bronchiolitis: predicting safe discharges from the emergency department. Pediatrics 2008;121:680-8.

6. Norwood A, Mansbach JM, Clark S, et al. Prospective multicenter study of bronchiolitis: predictors of an unscheduled visit after discharge from the emergency department. Acad Emerg Med 2010:17:376-82.

7. Marlais M, Evans J, Abrahamson E. Clinical predictors of admission in infants with acute bronchiolitis. Arch Dis Child 2011;96:648-52.

8. Kneyber MC, Moons KG, de Groot R, et al. Prediction of duration of hospitalization in respiratory syncytial virus infection. Pediatr Pulmonol 2002;33:453-7.

9. Moler FW, Ohmit SE. Severity of illness models for respiratory syncytial virus-associated hospitalization. Am J Respir Crit Care Med 1999;159:1234-40

10. Brown L, Reiley DG, Jeng A, et al. Bronchiolitis: Can objective criteria predict eligibility for brief hospitalization? CJEM 2003;5:239-44.

11. Rietveld E, Vergouwe Y, Steyerberg EW, et al. Hospitalization for respiratory syncytial virus infection in young children: development of a clinical prediction rule. Pediatr Infect Dis J 2006;25:201-7.

12. Vieira RA, Diniz EM, Ceccon ME. Correlation between inflammatory mediators in the nasopharyngeal secretion and in the serum of children with lower respiratory tract infection caused by respiratory syncytial virus and disease severity. J Bras Pneumol 2010;36:59-66.

13. Marguet C, Bocquel N, Benichou J, et al. Neutrophil but not eosinophil inflammation is related to the severity of a first acute epidemic bronchiolitis in young infants. Pediatr Allergy Immunol 2008;19:157-65.

14. Bont L, Heijnen CJ, Kavelaars A, et al. Peripheral blood cytokine responses and disease severity in respiratory syncytial virus bronchiolitis. Eur Respir J 1999;14:144-9.

15. Mansbach JM, Piedra PA, Laham FR, et al. Nasopharyngeal lactate dehydrogenase concentrations predict bronchiolitis severity in a prospective multicenter emergency department study. Pediatr Infect Dis J 2012;31:767-9.

16. Brand HK, Ferwerda G, Preijers F, et al. CD4+ T-cell counts and interleukin-8 and CCL-5 plasma concentrations discriminate disease severity in children with RSV infection. Pediatr Res 2013;73:187-93.

17. Mauri T, Coppadoro A, Bombino M, et al. Alveolar pentraxin 3 as an early marker of microbiologically confirmed pneumonia: a thresholdfinding prospective observational study. Crit Care 2014;18:562.

18. Sprong T, Peri G, Neeleman C, et al. Pentraxin 3 and C-reactive protein in severe meningococcal disease. Shock 2009;31:28-32.
19. Kim HS, Won S, Lee EK, et al. Pentraxin 3 as a clinical marker in children with lower respiratory tract infection. Pediatr Pulmonol 2016;51:42-8.

20. Appenzeller C, Ammann RA, Duppenthaler A, et al. Serum C-reactive protein in children with adenovirus infection. Swiss Med Wkly 2002;132:345-50.

21. Ruuskanen O, Putto $\mathrm{A}$, Sarkkinen $\mathrm{H}$, et al. C-reactive protein in respiratory virus infections. J Pediatr 1985;107:97-100.

22. Peltola V, Mertsola J, Ruuskanen O. Comparison of total white blood cell count and serum C-reactive protein levels in confirmed bacterial and viral infections. J Pediatr 2006;149:721-4.

23. Flood RG, Badik J, Aronoff SC. The utility of serum C-reactive protein in differentiating bacterial from nonbacterial pneumonia in children: a meta-analysis of 1230 children. Pediatr Infect Dis J 2008;27:95-9.

24. Pizzini C, Mussap M, Plebani M, et al. C-reactive protein and serum amyloid A protein in neonatal infections. Scand J Infect Dis 2000;32:229-35.

25. Huttunen T, Teppo AM, Lupisan S, et al. Correlation between the severity of infectious diseases in children and the ratio of serum amyloid A protein and C-reactive protein. Scand J Infect Dis 2003;35:488-90.

26. Nakayama T, Sonoda S, Urano T, et al. Monitoring both serum amyloid protein $\mathrm{A}$ and $\mathrm{C}$-reactive protein as inflammatory markers in infectious diseases. Clin Chem 1993;39:293-7.

27. Song Z, Cai L, Guo L, et al. Accumulation and expression of serum amyloid $\mathrm{P}$ component in human atherosclerotic lesions. Atherosclerosis 2010;211:90-5.

28. Skinner M, Vaitukaitis JL, Cohen AS, et al. Serum amyloid P-component levels in amyloidosis, connective tissue diseases, infection, and malignancy as compared to normal serum. J Lab Clin Med 1979;94:633-8.

29. Kemper C, Atkinson JP, Hourcade DE. Properdin: emerging roles of a pattern-recognition molecule. Annu Rev Immunol 2010;28:131-55.

30. Wirthmueller U, Dewald B. Thelen M, Schafer MK, Stover C, Whaley K, North J, Eggleton P, Reid KB, schwaeble WJ. Properdin, a positive regulator of complement activation, is released from secondary granules of stimulated peripheral blood neutrophils. $J$ Immunol 1997; 158:4444-51.

31. Cortjens B, de Boer OJ, de Jong R, et al. Neutrophil extracellular traps cause airway obstruction during respiratory syncytial virus disease. J Pathol 2016;238:401-11.

32. Jenne $\mathrm{CN}$, Wong $\mathrm{CH}$, Zemp FJ, et al. Neutrophils recruited to sites of infection protect from virus challenge by releasing neutrophil extracellular traps. Cell Host Microbe 2013;13:169-80.

33. Schejbel L, Rosenfeldt V, Marquart $\mathrm{H}$, et al. Properdin deficiency associated with recurrent otitis media and pneumonia, and identification of male carrier with Klinefelter syndrome. Clin Immunol 2009;131:456-62.

34. Späth PJ, Sjöholm AG, Fredrikson GN, et al. Properdin deficiency in a large Swiss family: identification of a stop codon in the properdin gene, and association of meningococcal disease with lack of the IgG2 allotype marker G2m(n). Clin Exp Immunol 1999;118:278-84.

35. Brand HK, de Groot R, Galama JM, et al. Infection with multiple viruses is not associated with increased disease severity in children with bronchiolitis. Pediatr Pulmonol 2012;47:393-400.

36. Frank $\mathrm{E}$, Hall $\mathrm{M}$, Trigg $\mathrm{L}$, et al. Data mining in bioinformatics using Weka. Bioinformatics 2004;20:2479-81.

37. Staley KG, Kuehni CE, Strippoli MP, et al. Properdin in childhood and its association with wheezing and atopy. Pediatr Allergy Immunol 2010;21:e787-e791.

38. Walsh P, Rothenberg SJ, O'Doherty S, et al. A validated clinical model to predict the need for admission and length of stay in children with acute bronchiolitis. Eur J Emerg Med 2004;11:265-72.

39. Prodhan P, Sharoor-Karni S, Lin J, et al. Predictors of respiratory failure among previously healthy children with respiratory syncytial virus infection. Am J Emerg Med 2011;29:168-73.

40. Bruun CF, Sanchez JC, Hochstrasser DF, et al. A two-dimensional electrophoretic study of serum amyloid $A$ and $C$-reactive protein in infants and children. Electrophoresis 1998;19:776-81. 